GSA DATA REPOSITORY 2009228

Supplementary electronic material for Geology article G25765

\title{
Earthquake histories and Holocene acceleration of fault displacement rates
}

\author{
Andrew Nicol ${ }^{1 *}$, John Walsh ${ }^{2}$, Vasiliki Mouslopoulou ${ }^{2}$, Pilar Villamor ${ }^{1}$ \\ ${ }^{1}$ GNS Science, PO Box 30368, Lower Hutt, New Zealand \\ ${ }^{2}$ Fault Analysis Group, School of Geological Sciences, University College Dublin, Belfield, \\ Dublin 4, Ireland \\ ${ }^{*}$ Corresponding Author (a.nicol@gns.cri.nz)
}

The following document contains material which is additional to, and in support of, the above manuscript. The manuscript provides an explanation for why Holocene displacement rates on dip-slip faults are often higher than rates measured over $>300 \mathrm{k} . \mathrm{y}$. and rates are similar for large ( $>4 \mathrm{~mm} / \mathrm{a})$ strike slip faults. Our discussion utilizes a global compilation of displacement rates for time intervals of approximately 0-10 k.y., 0-300 k.y. and 0->1 M.y. together with detailed analysis of active faults from the Taupo Rift in New Zealand. Displacement rates in Figure 1 of the article (see below) are presented in Table 1. Papers used to construct Table 1 are given below.
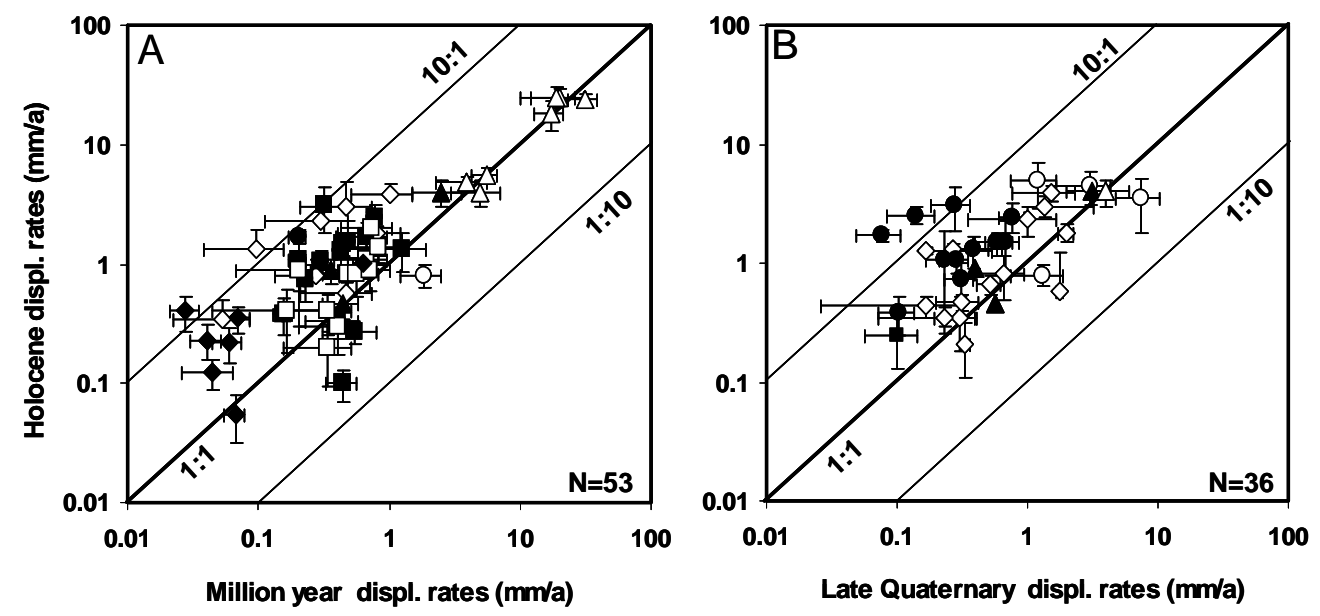

Figure 1 (from manuscript) Comparison of displacement rates averaged over the Holocene ( $\sim 10$ k.y.) with average values for the million year $(\geq 1$ M.y.) (A) and Late Quaternary $(\sim 300$ k.y.) (B) time intervals using a global data set of 64 faults. The names, locations (country and fault system), fault types, displacement rates (and their uncertainties) and data sources for the faults are given in supplementary Table 1 . The faults have a range of sizes, with lengths of 10 to $1300 \mathrm{~km}$ and total displacements of up to $\sim 480 \mathrm{~km}$, and slip rates of $\sim 0.01-30 \mathrm{~mm} / \mathrm{a}$. Symbols are as follows: unfilled triangle, strike-slip (San Andreas, Alpine, Dead Sea Transform, North Anatolian, Awatere, Sumatra and Itoilawa Shizuoku tectonic line faults); unfilled diamond, Taupo Rift (normal faults); unfilled circle, Corinth Rift (normal faults); unfilled square, Apennines (normal faults); filled triangle, New Zealand Hikurangi margin (reverse faults); filled diamond Taranaki Rift (normal faults); filled circle Wanganui Basin (reverse and normal faults) and filled square, Basin \& Range (normal faults). Regional strain rates are greater or less than $10^{-15}$ for unfilled and filled faults, respectively. Contours of the Holocene/Late Quaternary or M.y. displacement rate ratios are illustrated. 
Table 1 Summary of fault displacement rates plotted in Figure 1 of the manuscript together with data sources. For full references refer to the end of this supplement.

\begin{tabular}{|c|c|c|c|c|c|c|c|c|c|c|c|c|c|c|c|c|c|c|c|c|}
\hline \multirow{3}{*}{ Country } & \multirow{3}{*}{ Fault Name } & \multicolumn{6}{|c|}{ Holocene } & \multicolumn{6}{|l|}{$\begin{array}{l}\text { Late } \\
\text { Quaternary }\end{array}$} & \multicolumn{7}{|l|}{$\begin{array}{l}\text { Million } \\
\text { year }\end{array}$} \\
\hline & & Throw & Error & Age & Error & $\begin{array}{l}\text { Displ. } \\
\text { rate }\end{array}$ & Error & Throw & Error & Age & Error & $\begin{array}{l}\text { Displ. } \\
\text { rate }\end{array}$ & Error & Throw & Error & Age & Error & $\begin{array}{l}\text { Displ. } \\
\text { rate }\end{array}$ & Error & Source \\
\hline & & (m) & (m) & (ka) & (ka) & $(\mathbf{m m} / \mathbf{a})$ & $(\mathbf{m m} / \mathbf{a})$ & (m) & (m) & (ka) & (ka) & $(\mathbf{m m} / \mathbf{a})$ & $(\mathrm{mm} / \mathrm{a})$ & (m) & (m) & (ka) & (ka) & $(\mathbf{m m} / \mathbf{a})$ & $(\mathbf{m m} / \mathbf{a})$ & \\
\hline \multicolumn{21}{|l|}{$\begin{array}{l}\text { Fault Type: } \\
\text { Reverse }\end{array}$} \\
\hline New Zealand & Lachlan fault & 18 & 2 & 4.50 & 0.5 & 4.00 & 1.00 & 1100 & 200 & 350 & 50 & 3.14 & 1.02 & 2500 & 500 & 1000 & 200 & 2.50 & 1.00 & Barnes et al. (2002) \\
\hline New Zealand & Kidnappers fault & 16 & 2 & 18 & 2 & 0.89 & 0.21 & 106 & 13 & 270 & 30 & 0.39 & 0.09 & 360 & 40 & 1000 & 200 & 0.36 & 0.11 & Barnes \& Nicol (2004) \\
\hline New Zealand & Huangarua fault & 5.5 & 0.5 & 12 & 2 & 0.46 & 0.12 & 200 & 50 & 400 & 100 & 0.57 & 0.29 & 700 & 150 & 1600 & 150 & 0.44 & 0.14 & Nicol et al. (2002) \\
\hline New Zealand & Kapiti fault & 8 & 2 & 10.8 & 1 & 0.74 & 0.25 & 80 & 10 & 260 & 30 & 0.31 & 0.07 & 313 & 30 & 1350 & 40 & 0.23 & 0.03 & Lamarche et al. (2005), Nodder et al. (2007) \\
\hline New Zealand & Mascarin (total) fault & 16 & 2 & 6.5 & 1 & 2.46 & 0.69 & 200 & 20 & 260 & 30 & 0.77 & 0.17 & 1050 & 30 & 1350 & 40 & 0.78 & 0.05 & Lamarche et al. (2005), Nodder et al. (2007) \\
\hline New Zealand & Mascarin-2 fault & 2.5 & 0.5 & 6.5 & 1 & 0.39 & 0.14 & 27 & 5 & 260 & 30 & 0.10 & 0.03 & 210 & 30 & 1350 & 40 & 0.16 & 0.03 & Lamarche et al. (2005), Nodder et al. (2007) \\
\hline New Zealand & Moana fault & 11.5 & 1.5 & 10.8 & 1 & 1.07 & 0.24 & 73 & 10 & 260 & 30 & 0.28 & 0.07 & 400 & 30 & 1350 & 40 & 0.30 & 0.03 & Lamarche et al. (2005), Nodder et al. (2007) \\
\hline New Zealand & Okupe fault & 7 & 4 & 6.5 & 1 & 1.08 & 0.78 & 60 & 10 & 260 & 30 & 0.23 & 0.07 & 270 & 30 & 1350 & 40 & 0.20 & 0.03 & Lamarche et al. (2005), Nodder et al. (2007) \\
\hline New Zealand & Onepoto (total) fault & 16 & 2 & 10.8 & 1 & 1.48 & 0.32 & 150 & 30 & 260 & 30 & 0.58 & 0.18 & 590 & 30 & 1350 & 40 & 0.44 & 0.04 & Lamarche et al. (2005), Nodder et al. (2007) \\
\hline New Zealand & Otaheke North (total) fault & 14 & 3 & 10.8 & 1 & 1.30 & 0.40 & 100 & 20 & 260 & 30 & 0.38 & 0.12 & 570 & 30 & 1350 & 40 & 0.42 & 0.03 & Lamarche et al. (2005), Nodder et al. (2007) \\
\hline New Zealand & Rangitikei fault & 16.5 & 6.5 & 10.8 & 1 & 1.53 & 0.74 & 175 & 30 & 260 & 30 & 0.67 & 0.19 & 650 & 30 & 1350 & 40 & 0.48 & 0.04 & Lamarche et al. (2005), Nodder et al. (2007) \\
\hline New Zealand & Waitarere Total & 20 & 5 & 6.5 & 1 & 3.08 & 1.24 & 71 & 15 & 260 & 30 & 0.27 & 0.09 & 430 & 30 & 1350 & 40 & 0.32 & 0.03 & Lamarche et al. (2005), Nodder et al. (2007) \\
\hline \multicolumn{21}{|l|}{$\begin{array}{l}\text { Fault Type: } \\
\text { Normal }\end{array}$} \\
\hline New Zealand & Cape Egmont FZ & 14.4 & 2 & 14 & 2 & 1.03 & 0.29 & 84 & 20 & 224 & 30 & 0.38 & 0.14 & 2300 & 200 & 3700 & 200 & 0.62 & 0.09 & Nodder (1993) Nicol et al.(2005) \\
\hline New Zealand & Oaonui fault & 0.5 & 0.1 & 9 & 2 & 0.06 & 0.02 & & & & & & & 180 & 20 & 2700 & 200 & 0.07 & 0.01 & $\begin{array}{l}\text { Mouslopoulou et al.(2009), Fault Analysis } \\
\text { Group unpublished data (2008) }\end{array}$ \\
\hline New Zealand & Kiri fault & 4 & 0.5 & 10 & 2 & 0.40 & 0.13 & & & & & & & 83 & 15 & 3000 & 200 & 0.03 & 0.01 & $\begin{array}{l}\text { Mouslopoulou et al. (2009), Fault Analysis } \\
\text { Group unpublished data (2008) }\end{array}$ \\
\hline New Zealand & Ihaia fault & 3.5 & 0.2 & 10 & 2 & 0.35 & 0.09 & & & & & & & 190 & 30 & 2700 & 200 & 0.07 & 0.02 & $\begin{array}{l}\text { Mouslopoulou et al. (2009), Fault Analysis } \\
\text { Group unpublished data (2008) }\end{array}$ \\
\hline New Zealand & Kina fault & 2.2 & 0.3 & 10 & 2 & 0.22 & 0.07 & & & & & & & 160 & 30 & 2700 & 200 & 0.06 & 0.02 & $\begin{array}{l}\text { Mouslopoulou et al. (2009), Fault Analysis } \\
\text { Group unpublished data (2008) }\end{array}$ \\
\hline New Zealand & Rahotu fault & 1.6 & 0.2 & 13 & 2 & 0.12 & 0.03 & & & & & & & 120 & 40 & 2700 & 200 & 0.05 & 0.02 & $\begin{array}{l}\text { Mouslopoulou et al. (2009), Fault Analysis } \\
\text { Group unpublished data (2008) }\end{array}$ \\
\hline New Zealand & Inglewood fault & 3 & 0.5 & 13 & 2 & 0.23 & 0.07 & & & & & & & 150 & 30 & 3700 & 200 & 0.04 & 0.01 & $\begin{array}{l}\text { Hull (1996), Fault Analysis Group } \\
\text { unpublished data (2005) }\end{array}$ \\
\hline New Zealand & White Island fault & 35 & 5 & 15 & 2 & 2.30 & 0.64 & 300 & 100 & 300 & 100 & 1.00 & 0.67 & 400 & 100 & 1340 & 510 & 0.30 & 0.19 & $\begin{array}{l}\text { Lamarche et al. (2006), Nicol unpublished } \\
\text { data (2007) }\end{array}$ \\
\hline New Zealand & Rangitaiki fault & 35 & 3 & 9 & 1 & 3.89 & 0.77 & 460 & 30 & 300 & 100 & 1.53 & 0.61 & 1350 & 150 & 1340 & 510 & 1.01 & 0.50 & Taylor et al. (2004) \\
\hline
\end{tabular}




\begin{tabular}{|c|c|c|c|c|c|c|c|c|c|c|c|c|c|c|c|c|c|c|c|c|}
\hline \multirow{3}{*}{ Country } & \multirow{3}{*}{ Fault Name } & \multicolumn{6}{|c|}{ Holocene } & \multicolumn{6}{|l|}{$\begin{array}{l}\text { Late } \\
\text { Quaternary }\end{array}$} & \multicolumn{7}{|l|}{$\begin{array}{l}\text { Million } \\
\text { year }\end{array}$} \\
\hline & & Throw & Error & Age & Error & $\begin{array}{l}\text { Displ. } \\
\text { rate }\end{array}$ & Error & Throw & Error & Age & Error & $\begin{array}{l}\text { Displ. } \\
\text { rate }\end{array}$ & Error & Throw & Error & Age & Error & $\begin{array}{l}\text { Displ. } \\
\text { rate }\end{array}$ & Error & Source \\
\hline & & (m) & (m) & (ka) & (ka) & $(\mathbf{m m} / \mathbf{a})$ & $(\mathbf{m m} / \mathbf{a})$ & (m) & (m) & (ka) & (ka) & $(\mathbf{m m} / \mathbf{a})$ & $(\mathbf{m m} / \mathbf{a})$ & (m) & (m) & (ka) & (ka) & $(\mathbf{m m} / \mathbf{a})$ & $(\mathbf{m m} / \mathbf{a})$ & \\
\hline \multicolumn{21}{|l|}{$\begin{array}{l}\text { Fault Type: } \\
\text { Reverse }\end{array}$} \\
\hline New Zealand & Matata fault & 12 & 3 & 4 & 1.5 & 3.00 & 1.88 & 400 & 50 & 300 & 100 & 1.33 & 0.61 & 700 & 150 & 1500 & 500 & 0.47 & 0.26 & $\begin{array}{l}\text { Ota et al. (1988), Mouslopoulou et al. } \\
\text { (2008), }\end{array}$ \\
\hline New Zealand & Calyso faults & 16.5 & 4 & 20.5 & 2 & 0.81 & 0.27 & 200 & 30 & 300 & 100 & 0.67 & 0.32 & 370 & 50 & 1340 & 510 & 0.28 & 0.14 & Lamarche et al. (2006) \\
\hline New Zealand & Moutaki faults & 7.2 & 1.5 & 20.5 & 2 & 0.35 & 0.11 & 90 & 20 & 300 & 100 & 0.30 & 0.17 & & & & & & & Lamarche et al. (2006) \\
\hline New Zealand & Pukehoko fault & 9 & 2 & 20.5 & 2 & 0.44 & 0.14 & 50 & 10 & 300 & 100 & 0.17 & 0.09 & & & & & & & Lamarche et al. (2006) \\
\hline New Zealand & Tokata faults & 12 & 4 & 9 & 1 & 1.33 & 0.59 & & & & & & & 130 & 30 & 1340 & 510 & 0.10 & 0.06 & Lamarche et al. (2006) \\
\hline New Zealand & Ohiwa fault & 26 & 5 & 20.5 & 2 & 1.27 & 0.37 & 50 & 5 & 300 & 100 & 0.17 & 0.07 & & & & & & & Lamarche et al. (2006) \\
\hline New Zealand & Nukuhou fault & 27 & 5 & 20.5 & 2 & 1.32 & 0.37 & 80 & 10 & 300 & 100 & 0.27 & 0.12 & & & & & & & Lamarche et al. (2006) \\
\hline New Zealand & Braemer fault & 5.5 & 1 & 9.5 & 0.1 & 0.58 & 0.11 & 500 & 150 & 280 & 20 & 1.79 & 0.66 & 700 & 150 & 1500 & 500 & 0.47 & 0.26 & $\begin{array}{l}\text { Beanland et al. (1989), Mouslopoulou } \\
\text { (2006), Mouslopoulou et al. (2008) }\end{array}$ \\
\hline New Zealand & flt A & 7 & 2.5 & 17.5 & 2 & 0.34 & 0.16 & 70 & 20 & 300 & 30 & 0.23 & 0.09 & 80 & 20 & 1500 & 500 & 0.05 & 0.03 & Taylor et al. (2004) \\
\hline New Zealand & Paeroa fault & 17.2 & 2.4 & 9.55 & 0.1 & 1.80 & 0.27 & 550 & 50 & 280 & 20 & 1.96 & 0.32 & 1200 & 200 & 1500 & 500 & 0.80 & 0.40 & $\begin{array}{l}\text { Villamor \& Berryman (2001), Bibby pers } \\
\text { comm. (2002), Nicol unpublished data } \\
(2007)\end{array}$ \\
\hline New Zealand & Whirinaki fault & 6.3 & 1 & 9.55 & 0.1 & 0.66 & 0.11 & 155 & 20 & 300 & 30 & 0.52 & 0.12 & & & & & & & $\begin{array}{l}\text { Villamor \& Berryman (2001), Nicol } \\
\text { unpublished (2007) }\end{array}$ \\
\hline New Zealand & Ngakuru fault & 4.5 & 1 & 9.55 & 0.1 & 0.47 & 0.11 & 80 & 10 & 260 & 30 & 0.31 & 0.07 & & & & & & & $\begin{array}{l}\text { Villamor \& Berryman (2001), Nicol } \\
\text { unpublished (2007) }\end{array}$ \\
\hline New Zealand & Te Weta fault & 2 & 0.2 & 9.55 & 0.1 & 0.21 & 0.02 & 100 & 20 & 300 & 30 & 0.33 & 0.10 & & & & & & & $\begin{array}{l}\text { Villamor \& Berryman (2001), Nicol } \\
\text { unpublished (2007) }\end{array}$ \\
\hline New Zealand & Wairaka-North fault & 18.5 & 2 & 10.8 & 0.2 & 1.71 & 0.22 & 20 & 5 & 260 & 30 & 0.08 & 0.03 & 270 & 30 & 1350 & 40 & 0.20 & 0.03 & Lamarche et al. (2005), Nodder et al. (2007) \\
\hline New Zealand & Tangimoana fault & 27.5 & 4.5 & 10.8 & 0.2 & 2.55 & 0.46 & 36 & 10 & 260 & 30 & 0.14 & 0.05 & & & & & & & Lamarche et al. (2005), Nodder et al. (2007) \\
\hline Italy & Trasacco + Scurcola faults & 12.45 & 2 & 15 & 3 & 0.83 & 0.30 & & & & & & & 1455 & 200 & 3000 & 1000 & 0.49 & 0.23 & Roberts \& Michetti (2004) \\
\hline Italy & Liri + Carsoli faults & 16.5 & 2 & 15 & 3 & 1.10 & 0.35 & & & & & & & 2000 & 200 & 3000 & 1000 & 0.67 & 0.29 & Roberts \& Michetti (2004) \\
\hline Italy & $\begin{array}{l}\text { L'Aquila+Barete+Sulmona } \\
\text { faults }\end{array}$ & 20.7 & 2 & 15 & 3 & 1.38 & 0.41 & & & & & & & 2500 & 200 & 3000 & 1000 & 0.83 & 0.34 & Roberts \& Michetti (2004) \\
\hline Italy & Sella di Corno faults & 3 & 1 & 15 & 3 & 0.20 & 0.11 & & & & & & & 1000 & 200 & 3000 & 1000 & 0.33 & 0.18 & Roberts \& Michetti (2004) \\
\hline Italy & Rieti + Fiamignano faults & 12.45 & 2 & 15 & 3 & 0.83 & 0.30 & & & & & & & 1700 & 200 & 3000 & 1000 & 0.57 & 0.26 & Roberts \& Michetti (2004) \\
\hline Italy & Cassino South fault & 4.5 & 1 & 15 & 3 & 0.30 & 0.13 & & & & & & & 1200 & 200 & 3000 & 1000 & 0.40 & 0.20 & Roberts \& Michetti (2004) \\
\hline Italy & Fucino + Pescaseroli faults & 30 & 5 & 15 & 3 & 2.00 & 0.73 & & & & & & & 2200 & 200 & 3000 & 1000 & 0.73 & 0.31 & Roberts \& Michetti (2004) \\
\hline Italy & Leonessa fault & 6 & 1 & 15 & 3 & 0.40 & 0.15 & & & & & & & 1000 & 200 & 3000 & 1000 & 0.33 & 0.18 & Roberts \& Michetti (2004) \\
\hline
\end{tabular}




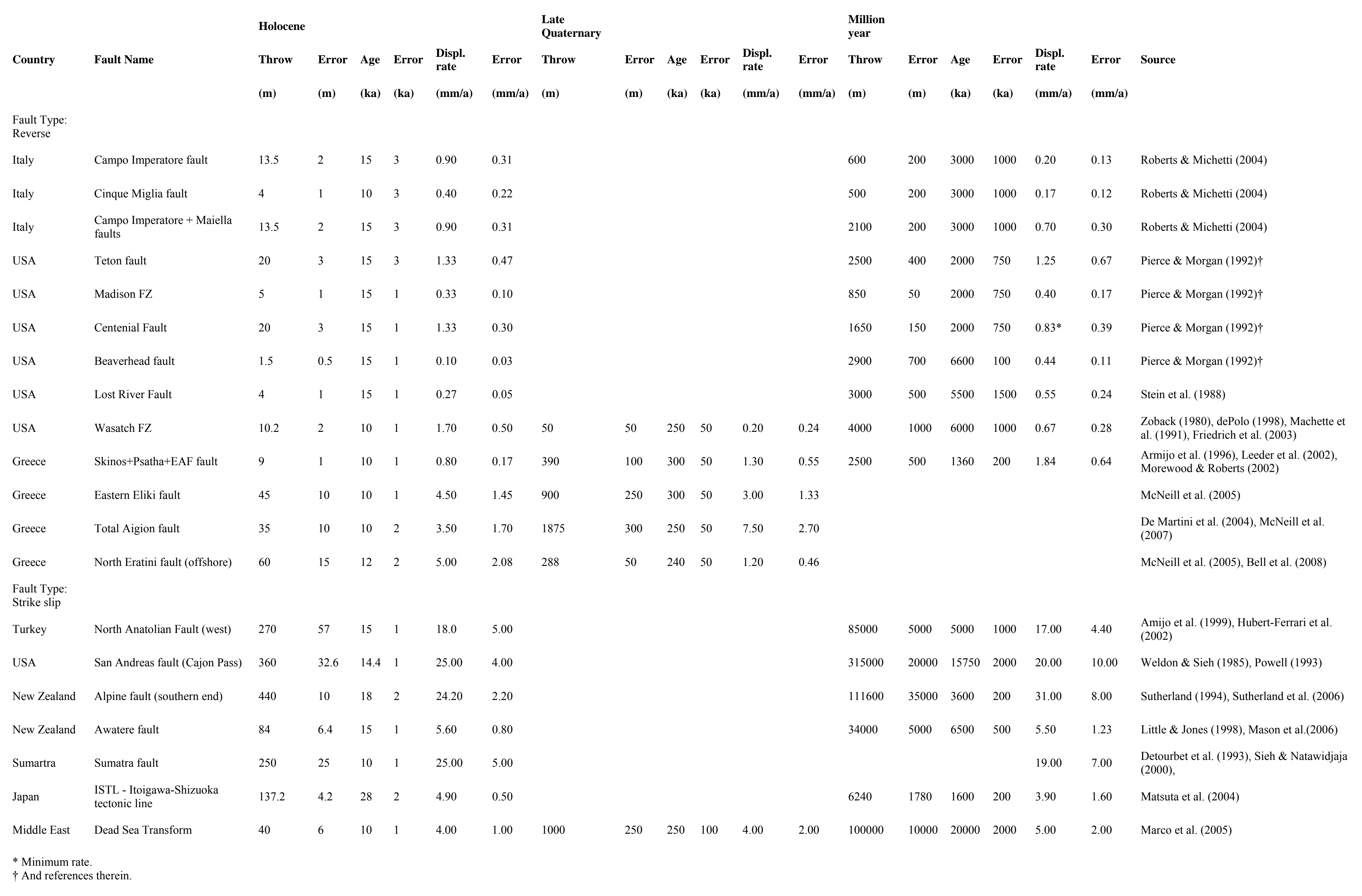




\section{References}

Anders, M.H., Geissman, J.W., Piety, L.A., Sullivan, J.T., 1989. Parabolic distribution of circumeastern Snake River Plain seismicity and latest Quaternary faulting: migratory pattern and association with the Yellowstone hotspot. Journal of Geophysical Research, v. 94, p. 1589-1621.

Armijo, R., Meyer, B., King, G.C.P., Rigo, A., Papanastasiou, D., 1996.

Quaternary evolution of the Corinth Rift and its implications for the Late Cenozoic evolution of the Aegean. Geophysical Journal International, v. 126, p. 11-53.

Barnes, P.M., Nicol, A., Harrison, T., 2002. Late Cenozoic evolution and earthquake potential of an active listric thrust complex, Hikurangi subduction margin, New Zealand. Geological Society American Bulletin, v.114, p. 1379-1405.

Barnes, P.M., Nicol, A. 2004. Active thrust triangle zone associated with structural inversion in a subduction forearc, eastern New Zealand. Tectonics, v. 23, TC1015, doi:10.1029/2002TC001449, 2004.

Beanland, S., Berryman, K.R., Blick, G.H., 1989. Geological investigations of the 1987 Edgecumbe earthquake. New Zealand Journal of Geology and Geophysics, v. 32, p. 73 91.

Bell, R.E., McNeill, L.C., Bull, J.M., Henstock, T.J., 2008. Evolution of the offshore western Gulf of Corinth. Geological Society of America Bulletin, v. 120, p. 156-178.

Berryman, K. R., Beanland, S., Wesnousky, S. 1998. Paleoseismicity of the Rotoitipakau Fault Zone, a complex normal fault in the Taupo Volcanic Zone, New Zealand. New Zealand Journal of Geology and Geophysics, v. 41, p. 449-465.

De Martini, P., Pantosti, D., Palyvos, N., Lemeille, F., McNeill, L., Collier, R., 2004. Slip rates of the Aigion and Eliki faults from uplifted marine terraces, Corinth Gulf, Greece. C. R. Geoscience, v. 336, p. 325-334.

dePolo, C.M., 1998. A reconnaissance technique for estimating the slip rate of normal slip faults in the Great Basin, and application to faults in Nevada, U.S.A.: Reno, University of Nevada. Ph.D. Thesis, 199 p.

Detourbet, C., Bellier, O., Sebrier, M., 1993. La caldera volcanique de Toba et le systeme de faille de Sumatra (Indonesia) vue par SPOT. Comptes Rendus de l'Acade'mie des Sciences. Se'rie II 316, p. 1439-1445.

Friedrich, A.M., Wernicke, B.P., Niemi, N.A., Bennett, R.A., Davis, J.L., 2003. Comparison of geodetic and geologic data from the Wasatch region, Utah, and implications for the spectral character of Earth deformation at periods of 10 to 10 million years: Journal of Geophysical Research, v. 108, B4, 2199, doi:10.1029/2001JB000682, 2003. 
Hubert-Ferrari, A., Armijo, R., King, G., Meyer, B., Barka, A., 2002. Morphology, displacement, and slip rates along the North Anatolian Fault, Turkey. Journal of Geophysical Research, v. 107, no. B10, 2235, doi:10.1029/2001JB000393,2002.

Hull, A., 1996. Past earthquake timing and magnitude along the Inglewood Fault, Taranaki, New Zealand. Bulletin of the New Zealand National society for Earthquake Engineering, v. 27, p. 155-162.

Lamarche, G., Proust, J.N., Nodder, S.D., 2005. Long-term slip rates and fault interactions under low contractional strain, Wanganui Basin, New Zealand. Tectonics, v. 24, TC4004, doi:10.1029/2004TC001699.

Lamarche, G., Barnes, P.M., Bull, J.M., 2006. Faulting and extension rate over the last 20,000 years in the offshore Whakatane Graben, New Zealand continental shelf. Tectonics, v. 25, TC4005, doi:10.1029/2005TC001886.

Leeder M.R., McNeill L.C., Collier, R.E.L., Portman, C., Rowe P.J., Andrews J.E., Gawthorpe, R.L., 2003. Corinth rift margin uplift: new evidence from Late Quaternary marine shorelines. Geophysical Research Letters, v. 30, p. 13-38.

Little, T. A., Jones, A., 1998. Seven million years of strike-slip and off-fault deformation on the Awatere Fault, South Island, New Zealand. Tectonics, v. 17, p. 285-302.

Machette, M.N., Personius, S.F., Nelson, A.R., Schwartz, D.P., Lund, W.R., 1991, The Wasatch fault zone, Utah-Segmentation and history of Holocene movement: Journal of Structural Geology, v. 13, p. 137-149.

McNeill L.C., Collier, R.E.L., Pantosti D., De Martini P.M., DAddezio G., 2005. Recent history of the Eastern Eliki Fault, Gulf of Corinth: geomorphology, palaeoseismology and impact on palaeoenvironments. Geophysical Journal International, v. 161, p. 154-166.

McNeill, L.C., Cotterill, C.J., Bull, J.M., Henstock, T.J., Bell, R. and Stefatos, A., 2007. Geometry and slip rate of the Aigion fault, a young normal fault system in the western Gulf of Corinth. Geology, v. 35, p. 355-358.

Marco, S., Stein, M., Agnon, A., 1996. Long-term earthquake clustering: A 50,000-yeal paleoseismic record in Dead Sea Graben. Journal of Geophysical Reaserch, v. 101, B3, 6179-6191, doi: 10.1029/95JB01587.

Marco, S., Rockwell, T.K., Heimann, A., Frieslander, U., Agnon, A., 2005. Late Holocene activity of the Dead Sea Transform revealed in 3D palaeoseismic trenches on the Jordan Gorge segment. Earth and Planetary Science Letters, v. 234, p. 189-205.

Mason, D.P.M., Little, T.A., Van Dissen, R.J., 2006. Rates of active faulting during late Quaternary fl uvial terrace formation at Saxton River, Awatere fault, New Zealand. Geological Society of America Bulletin, v. 118, p. 1431-1446; doi: 10.1130/B25961.1.

Matsuta, N., Ikeda, Y., Sato, H., 2004. The slip rate along the northern Itoigawa-Shizuoka tectonic line active fault system, central Japan. Earth Planets Space, v. 56, p. 1323-1330. 
Micarelli, L., Moretti, I., Daniel, J.M., 2003. Structural properties of rift-related normal faults: the case study of the Gulf of Corinth, Greece. Journal of Geodynamics, v. 36, p. 275-303.

Morewood, N.C., Roberts, G.P., 2002. Surface observations of active normal fault propagation: implications for growth. Journal of the Geological Society of London, $\mathrm{v}$. 159 , p. $263-272$.

Mouslopoulou, V., 2006. Quaternary geometry, kinematics and paleoearthquake history at the northern termination of the strike-slip North Island Fault System, New Zealand. PhD Thesis. Victoria University of Wellington, New Zealand.

Mouslopoulou, V., Nicol, A., Walsh, J.J., Beetham, D., Stagpoole, V., 2008. Quaternary temporal stability of a regional strike-slip and rift fault intersection. Journal of Structural Geology, v. 30, p. 451-463.

Mouslopoulou, V., Walsh, J.J., Nicol, A., 2009. Fault displacement rates on a range of timescales. Earth and Planetary Science Letters, v. 278, p. 186-197, doi:10.1016/j.eps1.2008.11.031.

Nicol, A., Van Dissen, R., Vella, P., Alloway, B., Melhuish, A., 2002. Growth of contractional structures during the last 10Ma, Hikurangi forearc, New Zealand. New Zealand Journal of Geology and Geophysics, v. 45, p. 365-385.

Nicol, A., Walsh, J.J., Berryman, K., Nodder, S., 2005. Growth of a normal fault by the accumulation of slip over millions of years. Journal of Structural Geology, v. 27, p. 327-342.

Nodder, S.D., 1993. Neotectonics of the offshore Cape Egmont Fault Zone, Taranaki Basin. New Zealand Journal of Geology and Geophysics, v. 36, p. 167-184.

Nodder, S.D., Lamarche, G., Proust, J.N., Stirling, M., 2007. Characterizing earthquake recurrence parameters for offshore faults in the low strain, compressional KapitiManawatu Fault System, New Zealand. Journal of Geophysical Research, v. 112, B12102, doi:10.1029/2007JB005019.

Ota, Y., Beanland, S., Berryman, K.R., Nairn, I.A. 1988. The Matata Fault: active faulting at the north-western margin of the Whakatane graben, eastern Bay of Plenty. New Zealand Geological Survey record, v. 35, p. 6-13.

Pierce, K. L., L. A. Morgan, 1992. The track of the Yellowstone hot spot: Volcanism, faulting, and uplift. Geological Society of America Memoir v. 179, p. 1-54.

Powell, R. E., 1993, Balanced palinspastic reconstruction of pre-late Cenozoic paleogeology, southern California: Geologic and kinematic constraints on evolution of the San Andreas fault system, in Powell, R. E.,Weldon, R. J., and Matti, J. C., eds., The San Andreas fault system: Displacement, palinspastic reconstruction, and geologic evolution: Geological Society of America Memoir, v. 178, p. 1-106. 
Roberts, G., Michetti, A., 2004. Spatial and temporal variations in growth rates along active fault systems: an example from Lazio-Abtuzzo, central Italy. Journal of Structural Geology, v. 26, p. 339-376.

Sieh, K., Natawidjaja, D., 2000. Neotectonics of the Sumatran fault, Indonesia, Journal of Geophysical Research, v. 105(B12), p. 28,295-28,326.

Stein, R. S., King, G. C., Rundle, J. B., 1988. The growth of geological structures by repeated earthquakes: 2. Field examples of continental dip-slip faults. Journal of Geophysical Research, v. 93, p. 13319-13331.

Sutherland, R., 1994. Displacement since the Pliocene along the southern section of the Alpine fault, New Zealand. Geology, v. 22, p. 327-330.

Sutherland, R., Berryman, K., Norris, R., 2006. Quaternary slip rate and geomporphology of the Alpine fault: Implications for kinematics and seismic hazard in southwest New Zealand. Geological Society of America Bulletin, v. 118, p. 464-474.

Taylor, S. K., Bull, J. M., Lamarche, G., Barnes, P. M. 2004. Normal fault growth and linkage in the Whakatane Graben, New Zealand, during the last 1.3 Myr. Journal of Geophysical Research, v. 109, B02408, doi:10.1029/2003JB002412.

Utah Quaternary Fault Parameters Working Group, 2005. Consensus preferred recurrence-interval and vertical slip-rate estimates: review of Utah paleoseismic-trenching data, Ed. William R. Lund, U.S. Geological Survey and Department of Natural Resources, Bulletin 134, 109 p., ISBN 1-55791-727-2, 6/05.

Villamor, P., Berryman, K., 2001. A late Quaternary extension rate in the Taupo Volcanic Zone, New Zealand, derived from fault slip data. New Zealand Journal of Geology and Geophysics, v. 44, p. 243-269.

Weldon, R.J., Sieh, K.E., 1985. Holocene rate of slip and tentative recurrence interval for large earthquakes on the San Andreas fault Cajon Pass, southern California. Geological Society of America Bulletin, v. 96, p. 793-812. 